\title{
Covariance approximation for fast and accurate computation of channelized Hotelling observer statistics
}

\author{
${ }^{\dagger}$ Paola Bonetto, ${ }^{\dagger}$ Jinyi Qi and ${ }^{\dagger}$ Richard M. Leahy \\ ${ }^{\dagger}$ Signal and Image Processing Institute, University of Southern California, Los Angeles, CA 90089-2564 \\ ${ }^{\ddagger}$ Dipartimento di Informatica e Scienze dell’Informazione, Unversity of Genoa, Italy \\ $\sharp$ Center for Functional Imaging, Lawrence Berkeley National Laboratory, Berkeley, CA 94720
}

\begin{abstract}
We describe a method for computing linear observer statistics for maximum a posteriori (MAP) reconstructions of PET images. The method is based on a theoretical approximation for the mean and covariance of MAP reconstructions. In particular, we derive here a closed form for the channelized Hotelling observer (CHO) statistic applied to 2D MAP images. We show reasonably good correspondence between these theoretical results and Monte Carlo studies. The accuracy and low computational cost of the approximation allow us to analyze the observer performance over a wide range of operating conditions and parameter settings for the MAP reconstruction algorithm.
\end{abstract}

\section{INTRODUCTION}

Image quality and the performance of image reconstruction algorithms can be assessed using quantitative and qualitative studies. The latter involve observers who evaluate the images from the perspective of a clinically relevant task such as lesion detection. The two major problems associated with psychophysical studies performed by human observers, namely high costs and subjective results, can be overcome by developing computer observers that reliably predict human performance. Methods for efficiently and accurately computing the statistics of these observers may prove very useful, especially when evaluating reconstructed image quality over a wide parameter space. These methods could be used to investigate changes in performance associated with variations in the parameters of the PET system, the source distribution, and the reconstruction method.

Here we develop a theoretical approximation for computing statistics of linear observers applied to PET images reconstructed using nonlinear MAP algorithms. The form of a linear computer observer for a binary lesion detection task is

$$
\boldsymbol{\lambda}_{\text {linear }}(\boldsymbol{g})=\left[\overline{\boldsymbol{g}}_{L}-\overline{\boldsymbol{g}}_{B}\right]^{\prime} \boldsymbol{K}^{-1} \boldsymbol{g},
$$

where $\overline{\boldsymbol{g}}_{L}$ and $\overline{\boldsymbol{g}}_{B}$ are the means of the two classes of images with and without lesions, respectively. The superscript ' denotes a matrix transpose and $\boldsymbol{K}$ is a matrix that depends on the specific form of the observer. For a non-prewhitened observer it is simply the identity matrix, while for a prewhitened observer, $\boldsymbol{K}$ is a covariance matrix that decorrelates the noise in the images [1]. A special form of (1) is the channelized Hotelling observer [2] in which the images $\boldsymbol{g}$ are filtered by frequency-selective channels that model properties of the human visual system. This model has been shown to successfully reflect human performance in detecting hot lesions in nuclear medicine images under a wide range of conditions $[2,3,4]$.

The Hotelling observer makes use of the covariances of the images. For linear algorithms this can be computed directly from the mean of the data by assuming a Poisson model. When the reconstruction estimator is nonlinear, analysis of the covariance is more difficult. Moreover, when the image is defined implicitly as the maximizer of a cost function, there will be no closed-form expression for the image covariance. Fessler [5] developed an approach to solving this problem by using truncated Taylor series approximations. In our previous work $[6,7]$ we adapted this approach to obtain approximate theoretical expressions for the covariance of MAP images as a function of the mean of the sinogram data. The approximate covariance is based on an assumption of local invariance in the response of the PET system and is computed for each voxel using Fourier transforms with only one data dependent parameter that can be obtained by a modified backprojection of the mean sinogram.

Here we use these approximations to compute the mean of the $\mathrm{CHO}$ observer statistic $\lambda(g)$. By expressing the related area under the ROC curve (AUC) directly as a function of the statistic [8] [9], we can describe the performance of the observer with a single expression that includes the features of the imaging device, the properties of the reconstruction algorithm, and the characteristics of the human visual system.

\section{THEORY}

We first review the mathematical aspects of the $\mathrm{CHO}$ and of the covariance approximation presented in [7]. Then, building on these results, we derive a closed form for the approximation of the statistic and of the relative AUC for the CHO.

As mentioned in section $\mathrm{I}$, the $\mathrm{CHO}$ is a special case of the linear observer (1) in which the reconstructions $\boldsymbol{g}$ are considered in a channel space defined by a matrix $\boldsymbol{U}$ whose rows represent the impulse response of frequency selective filters centered at the test location for the lesion [3]:

$$
\boldsymbol{\lambda}(\boldsymbol{g})=\left[\boldsymbol{U} \overline{\boldsymbol{g}}_{L}-\boldsymbol{U} \overline{\boldsymbol{g}}_{B}\right]^{\prime} \boldsymbol{K}_{U}^{-1} \boldsymbol{U} \boldsymbol{g},
$$

where we implicitly assume that the two types of images "No-lesion" and "Lesion" - are equally probable. $\boldsymbol{K}_{U}$ is the mean of the two covariance matrices for the two classes after transformation through the channels, i.e. it is the composite channel noise covariance matrix:

$$
\boldsymbol{K}_{U}=\frac{\boldsymbol{K}_{U L}+\boldsymbol{K}_{U B}}{2},
$$




$$
\boldsymbol{K}_{U i}=\left\langle\left[\boldsymbol{U} \boldsymbol{g}-\boldsymbol{U} \overline{\boldsymbol{g}}_{i}\right]\left[\left[\boldsymbol{U} \boldsymbol{g}-\boldsymbol{U} \overline{\boldsymbol{g}}_{i}\right]^{\prime}\right\rangle_{i} .\right.
$$

The notation \langle\rangle$_{i}$ specifies an avarage over the images belonging to class $i$.

The observer signal to noise ratio can be written as $[8,10]$

$$
S N R=\sqrt{\lambda\left(\overline{\boldsymbol{g}}_{L}-\overline{\boldsymbol{g}}_{B}\right)} .
$$

Moreover, under the assumption that $\boldsymbol{\lambda}(\boldsymbol{g})$ is Gaussian, the area under the ROC curve is related to the $\operatorname{SNR}[9,1]$ and can be written in terms of the observer statistic as

$$
A U C=\frac{1}{2}\left(1+\operatorname{erf}\left(\frac{\sqrt{\boldsymbol{\lambda}\left(\overline{\boldsymbol{g}}_{L}-\overline{\boldsymbol{g}}_{B}\right)}}{2}\right)\right) .
$$

The covariance approximation [5] of an implicitly defined estimator $\hat{x}$ is based on the first order Taylor expansion of the estimator itself:

$$
\begin{aligned}
\hat{\boldsymbol{x}}(\boldsymbol{y}) & \approx \hat{\boldsymbol{x}}(\overline{\boldsymbol{y}})+\nabla_{y} \hat{\boldsymbol{x}}(\overline{\boldsymbol{y}})(\boldsymbol{y}-\overline{\boldsymbol{y}}) \\
\operatorname{Cov}(\hat{\boldsymbol{x}}) & \approx \nabla_{y} \hat{\boldsymbol{x}}(\overline{\boldsymbol{y}}) \operatorname{Cov}(\boldsymbol{y})\left[\nabla_{y} \hat{\boldsymbol{x}}(\overline{\boldsymbol{y}})\right]^{\prime}
\end{aligned}
$$

where $\boldsymbol{y}$ is the data obtained from the unknown object $\boldsymbol{x}$, and $\overline{\boldsymbol{y}}$ is its mean.

The term $\nabla_{y} \hat{\boldsymbol{x}}(\overline{\boldsymbol{y}})$ can be derived from the objective function using the chain rule and the implicit function theorem [11]. For the specific case of Poisson emission data and MAP estimation it can be approximated as

$$
\nabla_{y} \hat{\boldsymbol{x}}(\overline{\boldsymbol{y}}) \approx[\boldsymbol{F}+\beta \boldsymbol{R}]^{-1} \boldsymbol{P}^{\prime} D\left[\frac{1}{\bar{y}_{i}}\right],
$$

where $\boldsymbol{F}=\boldsymbol{P}^{\prime} D\left[\frac{1}{\bar{y}_{i}}\right] \boldsymbol{P}$ is the Fisher information matrix when using a Poisson likelihood model, $\beta$ is the smoothing parameter of the MAP estimate, $\boldsymbol{R}$ is related to the second order derivative of the prior and $\boldsymbol{P}$ is the detection probability matrix $[5,6]$.

To find the term $\operatorname{Cov}(\boldsymbol{y})$ in (8), we note that the variability in the data is due to two factors, namely (i) the Poisson noise intrinsic to PET data, and (ii) any variability in the unknown image for which we will assume a locally stationary covariance $\Sigma_{x}$. It is straightforward to show that

$$
\operatorname{Cov}(\boldsymbol{y})=D\left[\bar{y}_{i}\right]+\boldsymbol{P} \Sigma_{x} \boldsymbol{P}^{\prime} .
$$

The combination of (9) and (10) into (8) leads to the final closed form for the approximate covariance for $\hat{\boldsymbol{x}}$ :

$$
\begin{aligned}
\operatorname{Cov}(\hat{\boldsymbol{x}}) & \approx[\boldsymbol{F}+\beta \boldsymbol{R}]^{-1} \boldsymbol{F}[\boldsymbol{F}+\beta \boldsymbol{R}]^{-1} \\
& +[\boldsymbol{F}+\beta \boldsymbol{R}]^{-1} \boldsymbol{F} \Sigma_{x} \boldsymbol{F}[\boldsymbol{F}+\beta \boldsymbol{R}]^{-1} .
\end{aligned}
$$

As shown in [12], this term can be computed on a voxel by voxel basis using Fourier transforms, by assuming the PET system response to be locally shift invariant, i.e.:

$$
\operatorname{Cov}_{j}(\hat{\boldsymbol{x}}) \approx \boldsymbol{Q}^{\prime} D\left[\frac{\kappa_{j}^{-2} \lambda_{i}(j)+\lambda_{i}^{2}(j) \tilde{\sigma}_{i}^{2}}{\left(\lambda_{i}(j)+\beta \kappa_{j}^{-2} \mu_{i}(j)\right)^{2}}\right] \boldsymbol{Q} \boldsymbol{e}_{j} .
$$

Here, $\boldsymbol{Q}$ and $\boldsymbol{Q}^{\prime}$ are the Kronecker form of the 2D DFT matrix and its inverse respectively; $\mu_{i}(j)$ and $\lambda_{i}(j)$ are related to the prior energy function in the MAP estimator and to the detection probability matrix respectively; $\kappa_{j}^{2}$ is the $(j, j)$ th element of the Fisher information matrix and it thus represents the aggregate uncertainty in the measurement rays that intersect the $j$ th pixel; $\tilde{\sigma}_{i}$ is the 2D Fourier transform of the covariance $\Sigma_{x}$ of the unknown object.

These equations are able to model a spatially variant detector response using a local-invariant approximation as we describe in [12]. However, they also assume a local stationarity in the covariance of the original image $\Sigma_{x}$. Clearly this assumption is not particularly well suited to the case of varying lesion sizes and activities. For this reason, the simulations presented in the following section assume a deterministic lesion but include random variation in the background activity in the form of a locally stationary Gaussian random field. If variability in the lesion is also to be included, then the contribution to the reconstructed image covariance from the source image covariance must be accounted for by specific computation in the spatial domain of the appropriate terms in (11). This will result in increased computation costs, but they will still remain orders of magnitude lower than that required for an equivalent Monte Carlo study.

In [7] we show how the covariance approximation can be modified to compensate for the nonlinear effect of the nonnegativity constraints typically used in MAP reconstruction and which are not taken into account when using the first order Taylor series expansion (8).

Approximation (11) is the key for explicitly computing the CHO statistic (2) in terms of the data. In fact, noting that in the case of MAP reconstruction the statistic's argument $\boldsymbol{g}$ is given by the estimate $\hat{\boldsymbol{x}}$, we have the following:

$$
\boldsymbol{K}_{U}=\boldsymbol{U} \boldsymbol{K} \boldsymbol{U}^{\prime}=\boldsymbol{U} \operatorname{Cov}(\hat{\boldsymbol{x}}) \boldsymbol{U}^{\prime} .
$$

The first order Taylor expansion (8) can be used again to approximate the mean of the reconstructions as the reconstruction of the noiseless data [5], and thus

$$
\overline{\hat{\boldsymbol{x}}}\left(\boldsymbol{y}_{L}\right)-\overline{\boldsymbol{x}}\left(\boldsymbol{y}_{B}\right) \approx \hat{\boldsymbol{x}}\left(\overline{\boldsymbol{y}}_{L}\right)-\hat{\boldsymbol{x}}\left(\overline{\boldsymbol{y}}_{B}\right) .
$$

We finally have

$$
\boldsymbol{\lambda}(\hat{\boldsymbol{x}}) \approx\left[\hat{\boldsymbol{x}}\left(\overline{\boldsymbol{y}}_{L}\right)-\hat{\boldsymbol{x}}\left(\overline{\boldsymbol{y}}_{B}\right)\right]^{\prime} \boldsymbol{U}^{\prime} \boldsymbol{U} \operatorname{Cov}(\hat{\boldsymbol{x}}(\boldsymbol{y})) \boldsymbol{U}^{\prime} \boldsymbol{U} \hat{\boldsymbol{x}}
$$

where $\operatorname{Cov}(\hat{\boldsymbol{x}}(\boldsymbol{y}))$ is given by (11).

Computing the AUC using (15) and (6) requires only two reconstructions from the mean of data, one each for the case with and without lesion, and a modified backprojection to compute $\kappa_{j}$ [13]. Compared to the Monte Carlo simulations that would otherwise be required to estimate (6), this represents a considerable advantage in terms of computational costs.

Equation (15) models in a single mathematical expression all properties of the imaging process - the PET system geometry and detector response, the reconstruction algorithm and human visual system. It allows us to isolate and study the effects of each component in a modular way and thus offers the potential for optimizing the system hardware and software for maximum lesion detectability, at least to the degree that the $\mathrm{CHO}$ model reflects true human observer performance. 


\section{Simulations AND RESUlts}

Two different kinds of simulations have been performed, one addressing the accuracy of the theoretical approximation through Monte Carlo studies and the other aimed at studying the performance of the theoretical approximation as a function of some of the key parameters that characterize PET images.

Monte Carlo analysis was performed on three sets of 100 2D images (128x128 pixels) reconstructed using a MAP algorithm for different values of the smoothing parameter $\beta$. Each data set consisted of sinograms computed from a 256x256 pixel phantom. The sinograms were computed using the realistic system model that we have developed for the CTI ECAT HR+ scanner [14]. Half of the sinograms in each data set were computed from a phantom without lesion, the other half from the phantom with a lesion at a fixed location with constant activity across a three pixel radius circle.

The first two data sets were obtained from the same chest phantom with the lesion located in the right lung. The first had a constant background to which the lesion was added in $50 \%$ of the cases. In the second data set, a different background was used in each phantom image by adding a smooth Gaussian random field to the lung as illustrated in Fig. 1. Each of the $144 \times 288$ element sinograms were scaled so that the mean number of counts generated was 200k and then realistic data generated using a Poisson pseudo-random number generator. Both data sets included attenuation effects but perfect attenuation correction factors were assumed known. Scatter was included only in the second set, in which a 5\% constant background was addded to the sinograms before generating the pseudo-Poisson data.

The last set of images was obtained from a brain phantom (Fig. 2), which includes more anatomical detail than the chest phantom. Again, a Gaussian random field was added to the white matter and the lesion was placed in $50 \%$ of the sinograms. A 5\% constant scatter was added to the sinograms and the total sinogram scaled to a mean of $400 \mathrm{~K}$ counts before generating pseudo-Poisson data.

For each of the three sets we computed the sample mean difference $\overline{\boldsymbol{g}}_{L}-\overline{\boldsymbol{g}}_{B}$ and the sample covariance matrix $\boldsymbol{K}_{U}^{-1}$ for the $\mathrm{CHO}$ observer for two different types of channel filters: DOG (difference Of Gaussian) and SQR (non-overlapping square filters), with three channels in each case. We then computed the performance of the observer as measured by the AUC, as defined in eq.(6).

The theoretical estimate of the AUC was obtained using equations (15) and (12). In this analysis we included the method proposed in [7] to compensate for the effects of the non-negativity constraints used in the MAP reconstructions. Note that instead of computing the mean difference $\overline{\boldsymbol{g}}_{L}-\overline{\boldsymbol{g}}_{B}$ between the noiseless reconstruction of the normal and abnormal phantom, as suggested in eq.(15), one could also estimate it as the convolution of the function representing the lesion with the local impulse response at the center of the lesion [12].

Comparisons of the two observers' performance, with reference to the three sets of images described above, are
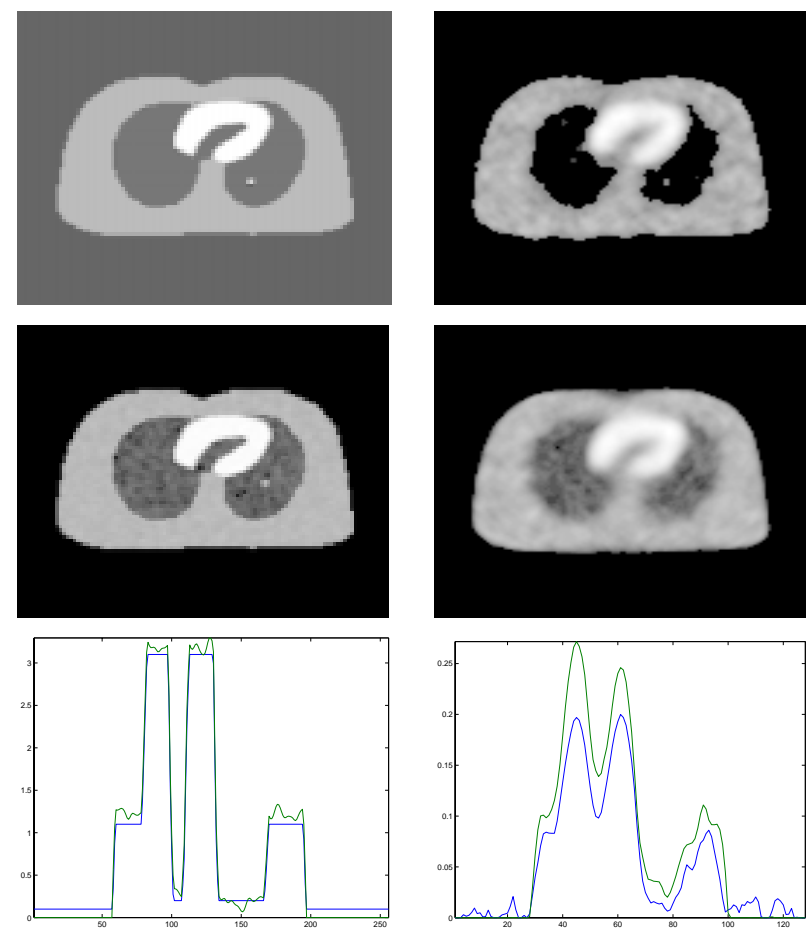

Figure 1: The transaxial chest plane phantom used in the simulations. The first row is related to the first study: the left image is the original abnormal phantom with values for lung:heart:soft tissue of 0.1:3.1:1.1. A 3 pixel radius lesion of intensity 2 is shown in the lower right lung. On the right is a MAP reconstruction for $\beta=100$. The second row refers to the second study: the left image is the original abnormal phantom with values for lung:heart:soft tissue of 0:3:1 to which we added a correlated Gaussian background with a mean of 0.2 . A 3 pixel radius lesion of maximum intensity 1.1 is shown in the lower right lung. On the right is a MAP reconstruction for $\beta=100$. The last row shows profiles of the $256 \times 256$ phantoms used in both studies (left), and of their $128 \times 128$ reconstructions for $\beta=100$ (right).
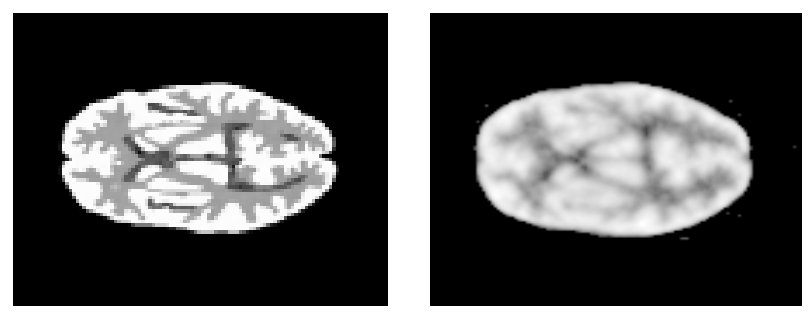

Figure 2: The transaxial brain plane phantom used in the simulations. Left: the original $256 \times 256$ abnormal phantom with values for white:grey:csf of 5:1:0, with a 3 pixel radius lesion of maximum intensity 0.9 in the right side of the brain, and with background variability of mean 0.2 . Right: a 128x128 MAP reconstruction for $\beta=10$.

summarized in Fig. 3, where the AUC values are plotted as a function of the MAP smoothing parameter $\beta$.

When no variability is present in the object, the two approaches show very good agreement. When background variability is included, the approximations used in the theoretical analysis have a greater influence on the final results, 


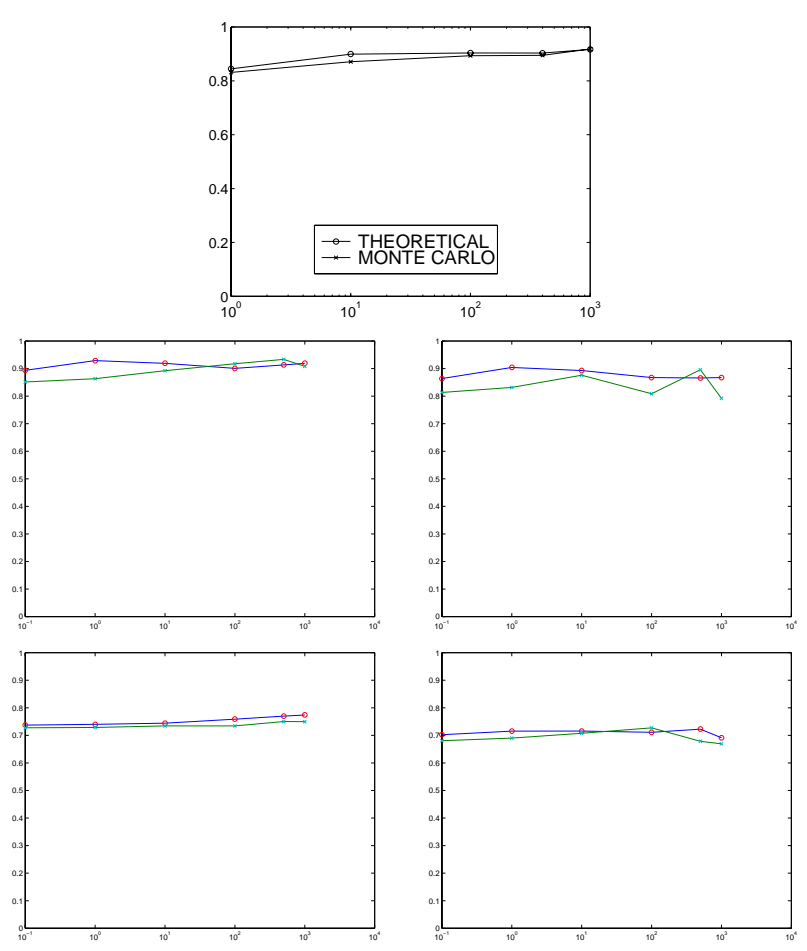

Figure 3: AUC values for the channelized Hotelling observer as a function of the smoothing parameter $\beta$, computed both by Monte Carlo simulations and by the theoretical approximation on the set of (1st row) chest phantom with no variability (DOG channels) (2nd row) chest phantom with background activity (left: DOG channels; right SQR channels) (3rd row) brain phantom with background activity (left: DOG channels; right SQR channels).

especially for the chest phantom. The major causes of the differences between Monte Carlo and the theoretical model are probably (i) the approximation of the mean difference with the difference of the noiseless normal and abnormal reconstructions, (ii) the assumption of space invariance used in computing the reconstructed image covariance, and (iii) the influence of the non negativity constraint on the accuracy of the covariance.

Differences are also due to the finite sample size of the sets used for the Monte Carlo simulations. In fact, the AUC values computed using sample statistics are affected by both variance and bias. To gain a deeper insight into these effects, we extended the first chest phantom study to 1000 images. We first addressed the problem of the variance, by applying the observer to several sets of $50+50$ reconstructions each, randomly choosen among the 1000 images. An example of the distribution of the values thus obtained is shown in figure 4. In this specific case, the distribution has a standard deviation of 0.031. We also plotted the AUC values as a function of the number of samples to examine changes in bias as a function of the number of images in the study. In the graph shown in figure 4 each AUC is obtained as the average of 250 AUC values. Because of the very high computational costs and storage space required, the image set is not large enough to allow one to pick completely independent sample sets, and thus the AUC values are correlated; nevertheless, the curve clearly shows a $1 / \mathrm{N}$ dependency on the number of samples. These observations are consistent with studies presented in [15].
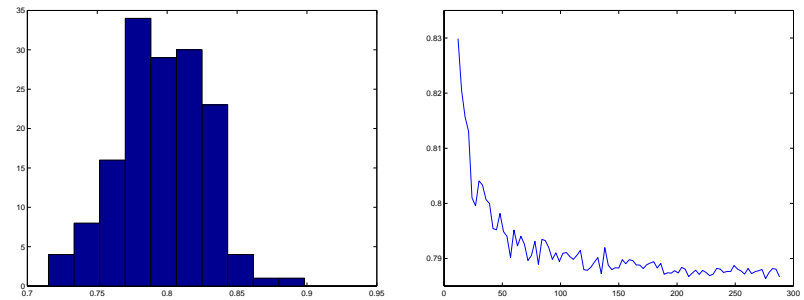

Figure 4: Accuracy of Monte Carlo simulations. Left: distribution of AUC values computed on 150 sets of 100 reconstructions each, randomly selected within a set of 1000 samples: standard deviation = 0.031. Right: AUC values as a function of set cardinality: each AUC value was obtained as the mean of 250 values.

One of the advantages of the theoretical expressions, is that we can study lesion detectability under various conditions without time consuming Monte Carlo reconstructions. As an example, we computed lesion detectability (SNR) as a function of the contrast of lesion to background, lesion size, and background noise (reflecting inhomogeneous tracer uptake). Fig. 5 shows the SNR for lesions with different contrast levels and sizes. The simulated lesion has a Gaussian shape. In general, SNR is linearly proportional to the contrast of the lesion, while it increases at a sub-linear rate as a function of size. As a result, for a fixed total lesion activity, it is easier to detect a high contrast small lesion than a low contrast large lesion. In Fig. 6 we plot SNR as a function of background
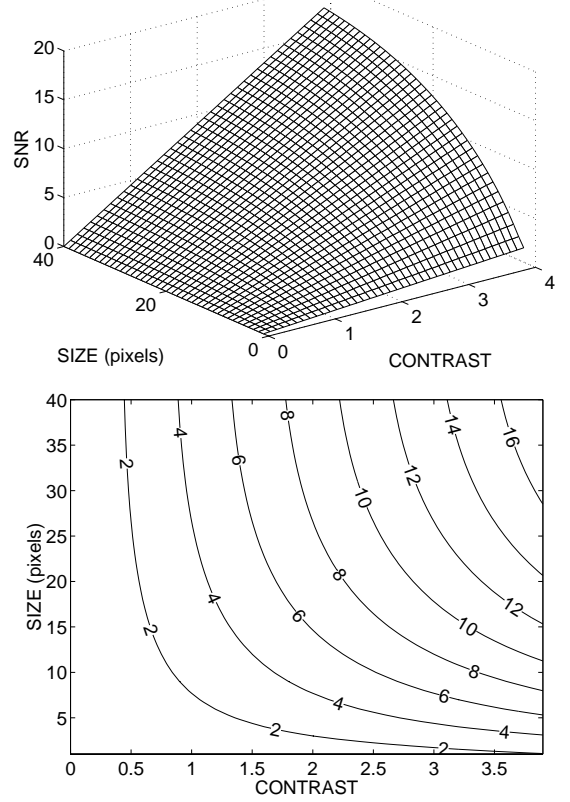

Figure 5: Surface and contour plot of the channelized Hotelling observer SNR (DOG channels) as a function of lesion contrast and size.

noise and lesion contrast. The noise level is determined by the variance of the Gaussian MRF used to model the inhomogeneity in tracer uptake. 

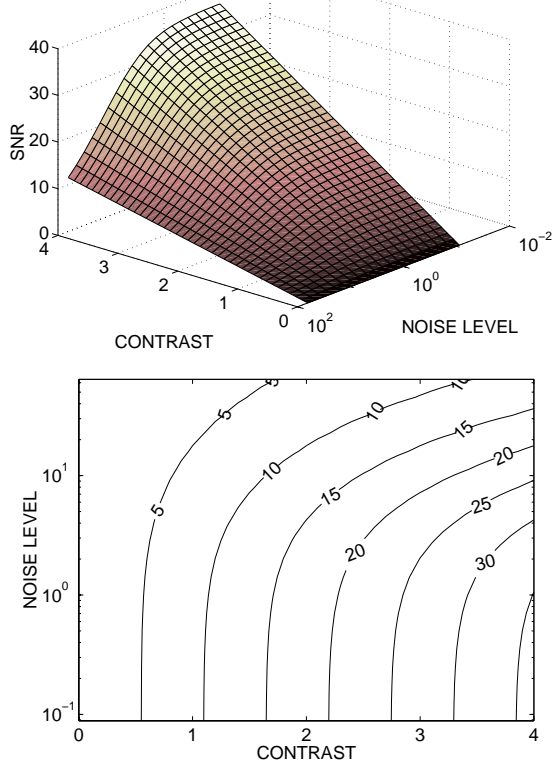

Figure 6: Surface and contour plot of the channelized Hotelling observer SNR (DOG channels) as a function of background noise level and lesion contrast.

\section{Conclusion}

Computer observers are becoming a popular alternative to human observers for performing psychophysical studies to assess image quality and performance of imaging systems and reconstruction algorithms. We have presented a theoretical approach to reducing the computational costs of linear observer statistics developed for the task of lesion detection in tomographic images. Studies using the channelized Hotelling observer for nonlinear MAP image reconstruction methods showed our model to be a fairly accurate and very low cost alternative to the traditional Monte Carlo studies. Using chest and brain phantoms we have shown the influence of smoothing parameter, lesion contrast, lesion size and background noise level on CHO performance. More generally, these closed form theoretical expressions allow us to compute observer performance over a large variety of parameter settings (including the system hardware configuration and reconstruction methods) and imaging features in order to study their effects on lesion detectability.

\section{ACKNOWLEDGMENTS}

This work was supported by the National Cancer Institute under Grant No. R01 CA59794 and Grant No. R01 CA56655 and by the U.S. Department of Health and Human Services under grant P01 HL25840.

\section{REFERENCES}

[1] H. H. Barrett, T. Gooley, K. Girodias, J. Rolland, T. White, and J. Yao, "Linear discriminants and image quality," Image Vision Comput., vol. 10, pp. 451-460, 1992.

[2] J. Yao and H. H. Barrett, "Predicting human performance by a channelized Hotelling observer model,"
Mathematical Method in Medical Imaging, SPIE, vol. 1768, pp. 161-168, 1992.

[3] M. A. King, D. J. de Vries, and E. J. Soares, "Comparison of the channelized Hotelling and human observers for lesion detection in hepatic SPECT imaging," SPIE, vol. 3036, pp. 14-20, 1997.

[4] C. K. Abbey, H. H. Barrett, and D. W. Wilson, "Observer signal-to-noise ratios for the ML-EM algorithm," SPIE, vol. 2712, 1996.

[5] J. A. Fessler, "Mean and variance of implicitly defined biased estimators (such as penalized maximum likelihood): Applications to tomography," IEEE Transactions on Image Processing, vol. 5, pp. 493-506, 1996.

[6] J. Qi and R. M. Leahy, "A theoretical study of the contrast recovery and variance of map reconstructions from PET data," IEEE Transactions on Medical Imaging, vol. 18, no. 4, pp. 293-305, April 1999.

[7] J. Qi and R. M. Leahy, "Fast computation of the covariance of MAP reconstructions of PET images," SPIE, vol. 3661, no. 1, pp. 344-355, February 1999.

[8] H. H. Barrett, "Objective assessment of image quality: effects of quantum noise and object variability," Journal of Optical Society of America A, vol. 7, pp. 1266-1278, 1990.

[9] A. E. Burgess, "Comparison of receiver operating characteristic and forced choice observer performance measurement methods," Med.Phys., vol. 22, pp. 643-655, 1995.

[10] K. J. Myers, "Addition of a channel mechanism to the ideal observer model," Journal of Optical Society of America A, vol. 4, pp. 2447-2457, 1987.

[11] J. Fessler and W. Rogers, "Spatial resolution properties of penalized-likelihood image reconstruction: Spaceinvariant tomographs," IEEE Transactions on Medical Imaging, vol. 5, pp. 1346-1358, 1996.

[12] J. Qi and R. M. Leahy, "Resolution and noise properties of map reconstruction for fully $3 \mathrm{~d}$ pet," International Meeting on Fully 3D Image Reconstruction in Radiology and Nuclear Medicine, June 1999.

[13] J. Qi, Bayesian Image Estimation for Fully 3D PET, Ph.D. thesis, University of Southern California, 1998.

[14] J. Qi, R. M. Leahy, C. Hsu, T. H. Farquhar, and S. R. Cherry, "Fully 3D bayesian image reconstruction for ECAT EXACT HR+," IEEE Transactions on Nuclear Science, vol. 45, no. 3, pp. 1096-1103, 1998.

[15] K. Fukunaga and R. R. Hayes, "Effects of sample size in classifier design," IEEE Transactions on Pattern Analysis and Machine Intelligence, vol. 11, pp. 873-885, 1989. 